\title{
Identification of Patients Requiring Adrenalectomy for Unilateral Adrenal Tumors Through a Dual Approach: ceCT and [18F]FDG-PET
}

Andrea Farolfi ( $\square$ andrea.farolfi3@gmail.com )

IRCCS Azienda Ospedaliero-Universitaria di Bologna

Elisa Maietti

University of Bologna

Federica Piperno

IRCCS Azienda Ospedaliero-Universitaria di Bologna

Pietro Coppolino

University of Catania

Guido Di Dalmazi

IRCCS Azienda Ospedaliero-Universitaria di Bologna

Guido Zavatta

IRCCS Azienda Ospedaliero-Universitaria di Bologna

Antonio De Leo

IRCCS Azienda Ospedaliero-Universitaria di Bologna

llaria Di Cintio

IRCCS Azienda Ospedaliero-Universitaria di Bologna

Stefano Fanti

IRCCS Azienda Ospedaliero-Universitaria di Bologna

Rita Golfieri

IRCCS Azienda Ospedaliero-Universitaria di Bologna

Alessandro Lambertini

Mittelrhein Community Clinic, Koblenz

Jennifer Malandra

IRCCS Azienda Ospedaliero-Universitaria di Bologna

Cristina Mosconi

IRCCS Azienda Ospedaliero-Universitaria di Bologna

Uberto Pagotto

IRCCS Azienda Ospedaliero-Universitaria di Bologna

Donatella Santini

IRCCS Azienda Ospedaliero-Universitaria di Bologna

\section{Saverio Selva}


IRCCS Azienda Ospedaliero-Universitaria di Bologna

\section{Valentina Vicennati}

IRCCS Azienda Ospedaliero-Universitaria di Bologna

\section{Cristina Nanni}

IRCCS Azienda Ospedaliero-Universitaria di Bologna

\section{Research Article}

Keywords: adrenal, adrenal tumor, CT, [18F]FDG PET/CT, positron emission tomography

Posted Date: November 8th, 2021

DOI: https://doi.org/10.21203/rs.3.rs-927691/v1

License: (9) (i) This work is licensed under a Creative Commons Attribution 4.0 International License. Read Full License 


\section{Abstract}

\section{Purpose}

Hormonal assessment (HA) and contrast-enhanced CT (ceCT) show insufficient sensitivity and specificity when staging unilateral adrenal lesions (ALs). We aimed at: 1) developing an imaging-based, i.e. ceCT and FDG-PET, diagnostic score able to discriminate adrenal tumors entailing adrenalectomy from those who need clinical monitoring, and 2) identifying a diagnostic flow-chart that allows clinicians to avoid unneeded diagnostic procedures and to address patients to the optimal management.

\section{Methods}

Retrospective single-center study assessing patients with unilateral $\mathrm{AL}$ and the following inclusion criteria: a) a four-phase ceCT; b) FDG-PET within one month of the ceCT; c) histopathology or a clinical follow-up of at least 24 months. Firstly, multivariate logistic regression analysis was employed to identify the predictors of adrenal tumors to treat surgically (AL-to-treat) and regression-based coefficients were used to develop a diagnostic score. Secondly, using cut-offs of predictor variables, a diagnostic flow-chart was defined.

\section{Results}

Forty-eight patients were enrolled (mean age 61 years), of whom 21/48 (44\%) had AL-to-treat. The remaining 27/48 (56\%) presented with $A L$ to follow-up only (i.e. benign). Maximum and minimum lesion diameter, Hounsfield units (HU) before contrast media injection and HU at the delayed phase (HUdelayed), relative and absolute washout, AL SUVmax, AL SUVmean, ratio SUVmax AL/SUVmax liver (adrenal-liver ratio) were associated with the presence of AL-to-treat (all $p<0.05)$. In multiple logistic regression analysis, SUVmax and HUdelayed showed to be significant predictors of AL-to-treat and were used to create a diagnostic score. ceCT parameters' cut-offs discriminating AL-to-treat surgically from AL-tofollow-up with 100\% PPV and NPV were first identified, finding 4/48 AL-to-treat and 15/48 ALs to followup. Applying the adrenal-liver ratio cut-off of 1.7 to the 29/48 remaining patients with uncertain AL management, for adrenal tumors we found an overall accuracy, sensitivity and specificity of $83 \%, 76 \%$ and $89 \%$, respectively, and a diagnostic flow-chart based on these results was proposed.

\section{Conclusion}

We developed a two-parameter imaging-based score that may be applied to predict adrenal tumors requiring adrenalectomy. Furthermore, a diagnostic flow-chart to stratify patients on the basis of the optimal management was proposed, thus guiding undefined unilateral adrenal lesions to FDG-PET imaging.

\section{Introduction}


Clinically silent unilateral adrenal masses detected by imaging studies performed for unrelated reasons, i.e. adrenal incidentalomas, have become a rather common finding in clinical practice. The vast majority are benign and non-functioning, but a minority represent functional or malignant tumors. A comprehensive review of the literature shows the prevalence of adrenal incidentalomas to be $2.3 \%$ at autopsy and $0.3-5.1 \%$ at abdominal computed tomography (CT) scan [1]. Up to $10 \%$ of patients with unilateral adrenal mass have autonomous secretion of adrenal hormones [1]. Most lesions are adrenocortical adenomas at histology, whereas the prevalence of adrenocortical carcinomas is relatively low. The risk of malignancy over time for masses defined as benign at diagnosis is estimated at about $1 / 1000$, even though $5-25 \%$ of masses increase in size during follow-up [1]. A biopsy of unclear adrenal masses to reach a definitive diagnosis is generally discouraged in the light of the chance of pheochromocytoma, whose puncture could cause severe complications. Therefore, laboratory tests and imaging are of utmost importance for the management of an adrenal mass as well as imaging tests that are used to calculate the risk of malignancy or, at least, to identify situations which need to be treated with surgery. The radiological definition of a unilateral adrenal lesion (AL) is the first key point to establish a correct diagnosis and address an appropriate management. Unenhanced density $<10$ Hounsfield units $(\mathrm{HU})$ at contrast-enhanced CT (ceCT) scan has been recommended as the gold standard to define a lipid-rich adrenal adenoma and safely rule out malignancy, considering the inverse relationship between tumor density and lipid content of the tumor [2]. On the contrary, lipid-poor lesions showing unenhanced density $\geq 10 \mathrm{HU}$ require further diagnostic procedures for a precise characterization. Employing ceCT scan with adrenal protocol ha/s been claimed as a useful method to discriminate between adenomas and non-adenomatous lesions [3]. Indeed, absolute (AWO) and relative washout (RWO), calculated from HU values derived from unenhanced, early contrast enhanced, and 15-minutes delayed ceCT scan, have been proposed as a reliable method to classify lipid-poor AL. Among tumors with unenhanced density $\geq 10 \mathrm{HU}$, AWO $>60 \%$ and RWO $>40 \%$ are currently employed to define a lipidpoor adenoma and to rule out pheochromocytoma and malignancy, including adrenocortical carcinoma and adrenal metastasis [4]. Despite the well-established role of ceCT scan, yet in the clinical practice unclear or border line cases are relatively frequent both regarding the laboratory profile and the radiological features. For instance, pheochromocytomas are rare catecholamine-secreting tumors with multiple clinical and imaging presentations. Pheochromocytomas may mimic other ALs, both benign and malignant, and several imaging appearances are described $[5,6]$. In these cases, positron emission tomography/computed tomography with $\left[{ }^{18} \mathrm{~F}\right] \mathrm{FDG}$ (FDG-PET) was proposed as a third level test for distinguishing benign from malignant ALs [7-9] with substantial sensitivity (74-100\%) and specificity (66-100\%) in patients with and without a oncological history. Nevertheless, a performing imaging algorithm to stratify patients into those who need a surgical approach (for different malignancies or longterm progressive diseases) and those who do not, could be used to make the correct clinical choice in this difficult clinical scenario. Moreover, a diagnostic flow-chart for unilateral AL characterization including ceCT with specific contrast phases and FDG-PET parameters has not been described yet.

The aim of this study was to develop an imaging-based, i.e. ceCT and FDG-PET, diagnostic score to discriminate patients with unilateral AL needing a surgical approach from those who need clinical 
monitoring. Furthermore, we aimed to develop a diagnostic flow-chart for unilateral AL, based on ceCT and FDG-PET parameters, for non-invasively addressing patients to the best management.

\section{Material And Methods}

\section{Study design and participants}

The cohort includes patients referred to the Unit of Endocrinology and Diabetes Prevention and Care for evaluation of unilateral AL from 2007 to 2018 and discussed within the multidisciplinary team for ALs, with the following inclusion criteria: a) four-phase ceCT scan; b) FDG-PET scan within one month from the ceCT; c) histopathology after adrenalectomy or a clinical follow-up of at least 24 months as reference standard. This retrospective study was approved by the institutional review board of the IRCCS Azienda Ospedaliero-Universitaria di Bologna (CE-AVEC).

\section{Imaging procedures}

CeCT was performed on unenhanced, arterial, venous and delayed (15 minutes) phases during the same examination. The adrenal ceCT protocol was composed of an early baseline acquisition before the injection of iodinated contrast agent, followed by a scan during the venous phase (70-80 seconds) and a delayed acquisition 15 minutes after infusion of contrast medium at a rate of $3-4 \mathrm{~mL} / \mathrm{s}$. For ceCT, 100$120 \mathrm{~mL}$ of $350 \mathrm{mg} \mathrm{l} / \mathrm{mL}$ low-osmolar contrast medium (iomeprol; lomeron 350, Bracco, Italy) was administered intravenously.

Whole body PET and low-dose CT scans without iodinated contrast medium were obtained one hour after the i.v. injection of $3.5 \mathrm{MBq} / \mathrm{Kg}$ of $18 \mathrm{~F}-2$-fluorodeoxyglucose (FDG-PET). A low dose CT scan was performed both for attenuation correction and to provide an anatomical map. An iterative 3-D ordered subsets expectation maximization method with two iterations and 20 subsets, followed by smoothing (with a 6-mm 3-D gaussian kernel) with CT-based attenuation, scatter and random coincidence event correction, was used to reconstruct the PET images. Patients fasted for 6 hours and insulin therapy was interrupted at least 6 hours before the examination in diabetic patients. All patients were positioned supine and acquired from the base of the skull to the mid thighs.

\section{Imaging interpretation}

$\mathrm{CeCT}$ dataset was reviewed independently by two radiologists with at least 5 years of experience in abdominal oncology. ALs were characterized by placing a ROI over two-thirds of the surface area of the AL for ceCT. As recommended by the current guidelines (2), we considered an adenoma as lipid rich when its attenuation at unenhanced CT was less than or equal to $10 \mathrm{HU}$. When attenuation at unenhanced CT was $>10 \mathrm{HU}$, the AWO and RWO at the contrast-enhanced CT scan were evaluated. The degrees of contrast enhancement and washout were based on the attenuation measurements of the adenoma in the unenhanced phase (i.e., unenhanced attenuation), contrast-enhanced venous phase (i.e., enhanced attenuation), and delayed phase (i.e., delayed attenuation). The AWO was calculated using the following formula: $A W O=[($ enhanced attenuation - delayed attenuation $) \times 100] /($ enhanced attenuation - 
unenhanced attenuation) [10]. AWO greater than $60 \%$ was considered RWO washout $=[($ enhanced attenuation - delayed attenuation) $\times$ 100] / enhanced attenuation [10]. An AL was considered as a lipidpoor adenoma when RWO was greater than $40 \%$. Axial maximum and minimum diameters, enhanced attenuation, unenhanced attenuation, delayed attenuation, absolute washout, and relative washout parameters were investigated for all the lesions.

The pattern of $\left[{ }^{18} \mathrm{~F}\right] \mathrm{FDG}$ uptake for ALs was visually classified by two nuclear medicine physicians with at least 5 years of experience in abdominal oncology in homogeneous and inhomogeneous. AL SUVmax and SUVmean were characterized by placing a volume-of-interest (VOI) over two-thirds of the surface area of the AL for FDG-PET. Additionally, ratio between AL SUVmax and liver SUVmax (adrenal-liver ratio), was calculated by placing a VOI of $50 \mathrm{~mm}$ of diameter in the right lobe of the liver.

\section{Hormonal assessment}

On the basis of clinical examination at presentation and imaging available at the multidisciplinary discussion, patients underwent hormonal assessment (HA), as recommended by the current guidelines (2). Briefly, patients underwent overnight $1 \mathrm{mg}$ dexamethasone suppression test with cortisol measurement in the morning, between 8:00 a.m. and 9:00 a.m., 8-9 hours after having taken $1 \mathrm{mg}$ of dexamethasone the previous night. Cortisol after dexamethasone administration $<50 \mathrm{nmol} / \mathrm{L}$ defined non-secreting adrenal tumors, whereas cortisol levels $>50 \mathrm{nmol} / \mathrm{L}$ defined autonomous cortisol secretion. Dexamethasone suppression test was performed in patients not taking corticosteroid treatments. Urinary metanephrines were measured in all patients, upon collection of 24-hours urine the previous day (patients received written instructions). Patients were advised to withdraw medications interfering with metanephrine determination for at least 4 weeks and to avoid catecholamine-containing foods for at least 7 days before urine collection. Aldosterone and renin were measured in hypertensive patients, after 2 hours of upright position, and after withdrawal of interfering medications during the previous 4 weeks, to calculate aldosterone-to-renin ratio (values below 1.2 were considered normal). Androgens and precursors (DHEA, DHEAs, androstenedione, testosterone, 170 Hprogesterone) were measured in all patients.

\section{Surgery}

Adrenalectomy was performed employing a transperitoneal laparoscopic technique with the patient in right or left lateral decubitus, with the use of three trocars on the left (optional fourth trocar) and four trocars on the right as a rule.

\section{Histopathology}

Formalin-fixed paraffin-embedded tissue blocks from phaeochromocytoma tumours underwent formal histopathologic analysis. Hematoxylin and eosin (H\&E) staining and immunohistochemistry (IHC) were performed on selected tumor blocks. All H\&E-stained slides of PCC specimens were reviewed by two experienced endocrine pathologists applying GAPP scoring system classification [11]. GAPP score was categorized as following 0-2: well differentiated (WD); 3-6: moderately differentiated (MD); and 7-10: poorly differentiated (PD), based on cut-points for risk stratification previously published. 


\section{Composite reference standard}

All patients were followed up for histopathologic analysis, imaging (ceCT, MRI, and PET) or HA acquired during clinical routine. Combination of (in descending priority) histopathologic analysis, imaging, and HA of at least 24 months follow-up was taken as composite reference standard. Unilateral ALs were classified into lesions requiring adrenalectomy (AL-to-treat), i.e. adrenal tumors, and namely adrenal carcinoma, lymphoma, leiomyosarcoma, pheochromocytoma and metastasis, and AL to follow-up only (AL-to-follow-up), namely adenoma, atypical adenoma, hemangioma, benign cyst and hyperplasia.

\section{Statistical analysis}

Continuous variables were summarized using mean and standard deviation (SD) if normally distributed, while median and interquartile range (IQR) were employed in case of a non-normal distribution; absolute and relative frequencies were used to describe categorical and dichotomous variables. Comparison between groups (AL-to-treat vs AL-to-follow-up) was performed using independent samples t-test for continuous variables with approximately normal distribution, Mann-Whitney test for skewed variables. Pearson's Chi-square test or Fisher's exact test were used to compare groups with respect to categorical variables.

\section{Imaging diagnostic score}

CeCT and FDG-PET parameters associated with diagnosis were included in a multiple logistic regression analysis with diagnosis as dependent variable ( 0 =AL-to-follow-up, $1=A L$-to-treat). To handle collinearity, a forward stepwise variable selection procedure was used. Results were reported as b-coefficients, odds ratios and $95 \%$ Confidence Interval $(95 \% \mathrm{Cl})$. The goodness of fit of the model was assessed using Hosmer \& Lemeshow test and the area under the ROC curve (AUC) was computed to determine its discriminative ability. The ceCT and FDG-PET parameters included in the multiple regression model were then categorized into quartiles to create a weighted predictive score. The weight assigned to each variable was equal to its regression coefficient divided by the coefficient in the regression with the smallest absolute value, rounded to the nearest integer number. The predictive score was obtained as a weighted sum.

\section{Diagnostic flow-chart}

ROC analysis of CeCT parameters predicting AL-to-treat surgically was performed. The cut-offs that maximized the negative and positive predictive values, i.e. the ability to identify AL patients to follow-up and AL patients to treat surgically, were then determined. These cut-offs were used to discriminate between patients with well-defined AL from those patients with undefined AL. In the subgroup with undefined AL, ROC analysis was applied to identify the FDG-PET parameter with the highest discriminative ability measured by the AUC. The best cut-off balancing sensitivity and specificity was then determined. 
A diagnostic flow-chart based on ceCT parameters' cut-offs followed by FDG-PET parameter's cut-off was defined. The discriminative ability of the overall algorithm was assessed in terms of sensitivity, specificity and overall accuracy (i.e. the percentage of patients correctly classified).

All analyses were performed using Stata statistical software version 15 (Stata Corp, College Station, TX) and the level of statistical significance was set to $a=0.05$.

\section{Results}

\section{Patient characteristics}

From our pathology database, 78 patients who had undergone a histopathological or clinical evaluation of a unilateral AL from 2007 to 2018 were identified. Of those, 51 patients with unilateral AL had undergone ceCT and FDG-PET within one month [median days 0 (range $-28 ;+31$ days)] and 48 patients had complete data and were used for the analyses (Figure 1). Thirty-eight patients were females (79\%), and 10 were males [mean age, 61 years \pm 13 years (range, 23-80 years)]. Diagnosis was reached from surgical resection in 27/48 (56\%) patients and clinical follow-up of at least 24 months in 21/48 (44\%). Most frequent diagnoses were adenoma in 22/48 (46\%), pheochromocytoma in 12/48 (25\%) and adrenal carcinoma in 5/48 (10\%). Based on GAPP score, pheochromocytomas were classified as welldifferentiated, moderately differentiated and poorly differentiated in 3/12 (25\%), 9/12 (75\%) and 0/12 $(0 \%)$, respectively. According to the composite reference standard, 21/48 (44\%) patients were diagnosed with unilateral AL-to-treat.

\section{ceCT and FDG-PET parameters}

Maximum and minimum axial lesion diameter, $\mathrm{HU}$ before contrast media injection, $\mathrm{HU}$ at the delayed phase (HU delayed), relative and absolute washout, were associated with the presence of a unilateral ALto-treat (all $p<0.05)$ (Table 1). The presence of a heterogeneous [18F]FDG uptake on PET images, SUVmax, SUVmean and SUVratio (adrenal-liver) were associated with unilateral AL-to-treat at final diagnosis (all $p<0.05)($ Table 1$)$. 
Table 1

Patient characteristics and comparisons of imaging findings between AL-to-treat and AL-to-follow-up.

\begin{tabular}{|c|c|c|c|c|}
\hline & $\begin{array}{l}\text { overall } \\
(n=48)\end{array}$ & $\begin{array}{l}\text { to follow-up } \\
(\mathrm{n}=27)\end{array}$ & $\begin{array}{l}\text { to treat } \\
\text { surgically } \\
(n=21)\end{array}$ & p-value \\
\hline Age (mean \pm SD) & $61 \pm 13$ & $60 \pm 12$ & $63 \pm 15$ & 0.455 \\
\hline Female, n (\%) & $38(79.2)$ & $20(74.1)$ & $18(85.7)$ & 0.325 \\
\hline \multicolumn{5}{|l|}{ AL size } \\
\hline $\begin{array}{l}\text { Maximum diameter, median [IQ } \\
\text { range] }\end{array}$ & $\begin{array}{l}25[19- \\
41.5]\end{array}$ & $22[18-29]$ & $33[23-54]$ & $0.014^{*}$ \\
\hline $\begin{array}{l}\text { Minimum diameter, median [IQ } \\
\text { range] }\end{array}$ & $21[14-31]$ & $19[13-22]$ & $26[19-42]$ & $0.022^{\star}$ \\
\hline \multicolumn{5}{|l|}{ ceCT parameters } \\
\hline Unenhanced $\mathrm{HU}$, mean $\pm \mathrm{SD}$ & $28.8 \pm 11.8$ & $25.3 \pm 12.5$ & $33.4 \pm 9.3$ & $0.017 *$ \\
\hline Arterial HU, mean \pm SD & $74.6 \pm 36.4$ & $72.6 \pm 40.4$ & $77.2 \pm 31.3$ & 0.664 \\
\hline Venous HU, mean \pm SD & $97.8 \pm 35.5$ & $91.0 \pm 38.6$ & $106.5 \pm 29.8$ & 0.135 \\
\hline Delayed HU, mean \pm SD & $64.4 \pm 26.3$ & $53.0 \pm 22.4$ & $79.1 \pm 23.9$ & $<0.001^{*}$ \\
\hline Absolute washout, mean \pm SD & $45.8 \pm 28.9$ & $52.9 \pm 32.6$ & $36.7 \pm 20.5$ & $0.042^{*}$ \\
\hline Relative washout, mean \pm SD & $33.5 \pm 21.2$ & $40.6 \pm 22.9$ & $24.4 \pm 14.8$ & $0.005^{\star}$ \\
\hline \multicolumn{5}{|l|}{ FDG-PET parameters } \\
\hline Inhomogeneous uptake, n (\%) & $26(54.2 \%)$ & $18(66.7 \%)$ & $8(38.1 \%)$ & $0.049 *$ \\
\hline SUVmax, median [IQ range] & $\begin{array}{l}5.5[4.0- \\
8.4]\end{array}$ & $4.6[3.7-6.4]$ & $7.0[5.4-11.1]$ & $0.001 *$ \\
\hline SUVmean, median [IQ range] & $\begin{array}{l}3.4[2.6- \\
5.0]\end{array}$ & $2.7[2.1-3.8]$ & $4.2[3.2-6.7]$ & $<0.001^{*}$ \\
\hline $\begin{array}{l}\text { SUVmax adrenal/liver, median [IQ } \\
\text { range] }\end{array}$ & $\begin{array}{l}1.4[1.1- \\
2.1]\end{array}$ & $1.1[0.9-1.7]$ & $2.0[1.7-2.4]$ & $<0.001^{*}$ \\
\hline $\begin{array}{l}\text { Notes. SD: standard deviation; IQ } r \\
\text { uptake value; * } p \text {-values }<0.05 \text {. }\end{array}$ & interquart & ge; HU: Ho & units; SUV: st & dized \\
\hline
\end{tabular}

\section{Imaging diagnostic score}

In the multiple logistic regression analysis including ceCT and FDG-PET parameters, HU delayed and SUVmax were found to be significant predictors of AL (Table 2). The model had an excellent discriminative ability (AUC=0.904, 95\%Cl: $0.820-0.987$ ) and a good fit to the data (Hosmer-Lemeshow 
test, $\mathrm{p}=0.822)$. HU delayed and SUVmax were categorized into quartiles that were assigned a score from 0 to 3. The description of quartiles is reported in Table 3. In the multiple regression model including these categorical variables, the regression coefficients were 1.49 for $\mathrm{HU}$ delayed and 1.21 for SUVmax, resulting in a weight of 1 for both (Table 2). The total score obtained as a weighted sum ranged from 0 to 6 . The percentage of patients with AL-to-treat diagnosis as a function of the predictive score is shown in Figure 2. A score $\leq 1$ has a NPV of $100 \%$, indicative of AL-to-follow-up. The likelihood of AL-to-treat increased with the increase in the score up to $100 \%$ for a score of 6 .

Table 2

Multiple logistic regression model to predict AL-to-treat vs AL-to-follow-up. Multiple model including continuous independent variables (Model 1) and multiple model including categorized into quartiles independent variables (Model 2).

\begin{tabular}{|c|c|c|c|c|c|c|}
\hline & Model 1 & & & Model 2 & & \\
\hline & $\begin{array}{l}\text { b } \\
(95 \% \mathrm{Cl})\end{array}$ & $\begin{array}{l}\text { OR } \\
(95 \% \mathrm{Cl})\end{array}$ & p-value & $\begin{array}{l}\text { b } \\
(95 \% \mathrm{Cl})\end{array}$ & $\begin{array}{l}\text { OR } \\
(95 \% \mathrm{Cl})\end{array}$ & p-value \\
\hline Delayed HU & $\begin{array}{l}0.07 \\
(0.02-0.12)\end{array}$ & $\begin{array}{l}1.07 \\
(1.02-1.13)\end{array}$ & $0.003^{\star}$ & $\begin{array}{l}1.49 \\
(0.51-2.47)\end{array}$ & $\begin{array}{l}4.44 \\
(1.66-11.82)\end{array}$ & $0.003^{\star}$ \\
\hline SUVmax & $\begin{array}{l}0.60 \\
(0.15-1.05)\end{array}$ & $\begin{array}{l}1.82 \\
(1.16-2.85)\end{array}$ & $0.009 *$ & $\begin{array}{l}1.21 \\
(0.37-2.04)\end{array}$ & $\begin{array}{l}3.34 \\
(1.45-7.71)\end{array}$ & $0.005^{\star}$ \\
\hline
\end{tabular}

Table 3

HU delayed and SUVmax quartiles

\begin{tabular}{|llll|}
\hline Quartiles & Delayed HU & SUVmax & score \\
\hline Q1 & $5-46$ & $2.6-4$ & 0 \\
\hline Q2 & $47-59$ & $4.1-5.4$ & 1 \\
\hline Q3 & $60-82$ & $5.5-8.4$ & 2 \\
\hline Q4 & $\geq 83$ & $\geq 8.5$ & 3 \\
\hline
\end{tabular}

\section{FDG-PET with low-dose CT}

In a secondary analysis including only parameters derived from a single FDG-PET examination made of a low-dose CT without contrast medium injection (lesion diameter, HU before contrast media injection, SUVmax, SUVmean and SUV ratio), only adrenal-liver SUV ratio was found to be a significant predictor of diagnosis (AL-to-treat vs. AL-to-follow-up) in the multiple logistic regression analysis. Its discriminative 
ability was lower (AUC=0.809, 95\% Cl: $0.686-0.931)$ than that of the multiple model including $\mathrm{HU}$ delayed and SUVmax (see Supplemental Figure 1).

\section{Diagnostic flow-chart}

ROC analysis was applied to identify diagnostic cut-offs on ceCT parameters (Supplemental Figures 2-5). $\mathrm{HU}$ delayed $<47$ and relative washout $>50 \%$ identified AL-to-follow-up, with a NPV $=100 \%$ and PPV of $58.3 \%$ and $53.8 \%$ respectively (Table 4). Conversely HU delayed $>100$ identified AL-to-treat with a PPV $=100 \%$ and NPV $=61.4 \%$ (Table 4). No other cut-off leading to a $100 \%$ NPV or PPV was identified among the other ceCT parameters. Using ceCT parameters cut-offs, 4 patients with AL-to-treat and 15 patients with AL-to-follow-up were identified and 29 patients with uncertain AL management were thus eligible for FDG-PET assessment. In this subgroup, adrenal-liver SUV ratio was the FDG-PET parameter with the highest discriminative ability (AUC=0.738, 95\% Cl: $0.540-0.935)$ (Supplemental Table 2) and SUV ratio $>1.7$ resulted the optimal cut-off with $70.6 \%$ sensitivity and $75 \%$ specificity (Table 4 ). Based on these results, a diagnostic flow-chart designed to address patients to surgical treatment or follow-up was defined (Figure 3). Applying the ceCT assessment followed by the SUVmax cut-off to those remaining patients with uncertain management, the diagnostic flow-chart showed an overall accuracy of $83.3 \%$, sensitivity of $76.2 \%$ and specificity of $89 \%$ for AL-to-treat (Table 4 ).

Table 4

Diagnostic accuracy of ceCT and FDG-PET parameters in predicting AL-to-treat diagnosis.

\begin{tabular}{|llllll|}
\hline & PPV & NPV & sensitivity & specificity & accuracy \\
\hline HU delayed $>100$ & $100 \%$ & $61.4 \%$ & $19.0 \%$ & $100 \%$ & $64.6 \%$ \\
\hline HU delayed $<47$ & $58.3 \%$ & $100 \%$ & $100 \%$ & $44.4 \%$ & $68.8 \%$ \\
\hline Relative washout $>50 \%$ & $53.8 \%$ & $100 \%$ & $100 \%$ & $33.3 \%$ & $62.5 \%$ \\
\hline SUV ratio $>1.7 \S$ & $80.0 \%$ & $64.3 \%$ & $70.6 \%$ & $75 \%$ & $72.4 \%$ \\
\hline Diagnostic flow-chart & $84.2 \%$ & $82.8 \%$ & $76.2 \%$ & $88.9 \%$ & $83.3 \%$ \\
\hline
\end{tabular}

Notes: $\S$ among patients with undefined AL diagnosis after ceCT assessment $(n=29)$.

\section{Laboratory Tests}

HA is shown in Supplemental Table 1 and was available for 30/48 (63\%) of patients. Among those, $26 / 30(87 \%)$ had at least one altered test result. Within the group with a diagnosis of adenoma at the composite reference standard $(n=22), 16 / 22(73 \%)$ underwent HA and of those 14/16 (88\%) were secerning adenoma. Considering the heterogeneity of diagnosis within the group to treat surgically, only norepinephrine- $U(n=23)$ was associated with the presence of an adrenal tumor on composite reference standard $(p=0.031)$. ROC analysis was applied to identify a diagnostic cut-off for norepinephrine-U (Supplemental Figure 6). Values $>70 \mathrm{mg} / 24 \mathrm{~h}$ identified AL-to-treat with a PPV $=100 \%$ and NPV $=76.0 \%$. All 
the patients with abnormal results for one of the following: norepinephrine- $U(n=3)$, dopamine $U(n=2)$, metanephrine- $U(n=2)$ and normetanephrine- $U(n=2)$ had a diagnosis of AL-to-treat.

\section{Discussion}

All patients with unilateral AL should be screened for malignancy. For incidentalomas with a benign appearance on imaging, international guidelines suggest a repeat imaging study at 12 months after initial discovery [2]. The rationale is that several malignant lesions can grow in this time, leading to earlier intervention. In principle, the appropriateness and suitability of surgery should be guided by the likelihood of malignancy, the presence and degree of hormone excess, age, general health and patient preference. Despite the well-established role of imaging, still unclear or borderline unilateral ALs are found, both regarding the hormonal profile and the radiological features. Molecular imaging employing FDG-PET has been proposed for unilateral AL characterization and a systematic review including 29 studies (2421 patients) found a pooled sensitivity for FDG-PET of $91 \%(95 \% \mathrm{Cl}: 88 \%-94 \%)$ and a pooled specificity of 91\% (95\% Cl: $87 \%$ - 93\%) for AL characterization [12]. In the current study we enrolled 48 patients with unilateral AL who had undergone ceCT and FDG-PET before adrenalectomy or 24 months follow-up. On the basis of the composite reference standard, approximately $44 \%$ of patients had a unilateral adrenal tumor requiring adrenalectomy. Most importantly, regarding the final diagnosis, the cohort of patients was balanced, with about $56 \%$ of benign diagnosis (e.g. adenoma, hyperplasia), $25 \%$ of pheochromocytoma and $19 \%$ of adrenal carcinoma. Here we present a dual approach to the management of unilateral ALs. Firstly an imaging diagnostic score based on ceCT and FDG-PET parameters and, secondly, a diagnostic flow-chart for optimizing patient management and avoiding unneeded FDG-PET procedure. FDG-PET cut-off values have been investigated in previous publications for ALs characterization [13-16]. Tessonnier and colleagues reported the value of FDG-PET in the diagnosis of 41 indeterminate ALs (29\% malignant) [13]. They showed that the use of an adrenal-liver ratio SUVmax was more accurate than visual assessment in the distinction between benign and malignant ALs, with an optimal threshold value of 1.8. Altinmakas et al retrospectively enrolled 59 patients with $61 \mathrm{AL}$ and FDG-PET before histologic evaluation, of those $88 \%$ were malignant lesions (44\% metastasis) [14]. They found a threshold for adrenal-liver ratio SUVmax of 0.83 in discriminating malignant ALs, which yielded accuracy of $87 \%$ and sensitivity of $98 \%$. Moreover, when combined with non-contrast CT density of the AL, the diagnostic performance of both individual FDG-PET parameters increased, both combinations yielding essentially the same accuracy of $93 \%$ and AUCs of 0.81 . However, in this surgical cohort of patients only few of them had benign AL, no pheochromocytoma was found and injection of iodinated contrast medium with multiphase CT was not performed. In our study, considering the maximum lesion diameter, unenhanced $\mathrm{HU}$ and adrenal-liver SUVmax ratio, an AUC of 0.809 was found, comparable to the study by Altinmakas and colleagues. FDG-PET made up of a low-dose unenhanced CT part of the scan could thus be a feasible one-stop-shop examination for assessing unilateral ALs in patients where iodinated contrast medium is contraindicated or when incidentally detected firstly by FDG-PET, even if the AUC is lower than the one found for FDG-PET and multi-phase ceCT $(A U C=0.904 ; p=0.110)$. In a prospective multicenter study on 87 adrenal masses in non-cancer 
patients, histology was obtained in $74 \%$ of cases and 12 -months follow-up was performed in the remaining patients [15]. Overall, $83 \%$ were benign $\mathrm{AL}, 2 \%$ were pheochromocytoma and tumors diagnosed with uncertain malignant potential on final pathology were considered as benign. The optimal threshold value of the adrenal-liver ratio SUVmax for malignancy was 1.5 with sensitivity, specificity, positive predictive value, negative predictive value, and accuracy of $86.7 \%, 86.1 \%, 56.5 \%, 96.9 \%$, and $86.2 \%$, respectively. Nevertheless, there is clinical need for a simple and practical diagnostic algorithm able to discriminate patients to follow-up from patients to treat surgically. We developed a two-parameters imaging score based on ceCT delayed phase and FDG-PET SUVmax able to discriminate, in a cohort made up of benign and malignant lesions including pheochromocytoma, patients with adrenal tumors to treat surgically from patients to follow-up with an AUC of 0.904 , slightly higher than the AUC of 0.868 found by Guerin et al. Based on these results, a risk score was proposed, showing an excellent predictive value for scores of 5-6. On the other hand, a simple diagnostic flow-chart to address patients to the most accurate management in order to avoid unneeded diagnostic procedures is missing for ALs. Hormonal assessment together with ceCT discriminate most of patients with a unilateral $A L$ and only patients with an uncertain diagnosis would be referred to FDG-PET especially in a cost-effectiveness rationale. Indeed, ESE and ENSAT guidelines suggest to perform non-contrast CT and HA when assessing AL risk of malignancy [2]. If the $A L$ is indeterminate on non-contrast CT and the result of the HA do not indicate significant hormone excess, there are three options that should be contemplated, namely immediate additional imaging (i.e. MRI, ceCT and FDG-PET), interval imaging in 6-12 months or surgery, guidelines say. However, there is lack of evidence and studies reporting direct comparison of these different imaging modalities. We calculated cut-offs for ceCT parameters to distinguish patients with defined diagnosis from patients with uncertain management. Applying the FDG-PET adrenal-liver ratio SUVmax cut-off of 1.7 to those remaining patients with uncertain management, we found an overall accuracy of $83 \%$, sensitivity of $76 \%$ and specificity of $89 \%$ for adrenal tumors to treat surgically and a manageable diagnostic flow-chart was designed accordingly.

Summarizing, we proposed two distinct tools able to efficiently support clinicians, radiologists and nuclear medicine physicians when assessing ALs and to optimize patient management.

This study has a number of strengths in comparison to prior studies evaluating ceCT and FDG-PET for unilateral ALs characterization. These include the multidisciplinary approach to included patients, ceCT protocol made up of four phases, short timeframe between ceCT and FDG-PET, i.e. one month, implementation of independent readers, and lesions validation by adrenalectomy or clinical follow-up of at least 24 months. Lastly, a reasonably narrow dimensional range of the AL compared to previous studies. However, major limitations are the retrospective design, the imaging approach based solely on ceCT and FDG-PET without including MRI as well as the lack of a systematic hormonal screening in all patients, and the small sample size. In addition, the imaging-based algorithm and the diagnostic flowchart are not validated in an independent sample. Therefore, further prospective evidence is needed to externally validate both the predictive score and the diagnostic flow-chart in larger cohorts of patients. 


\section{Conclusion}

This study shows that combined contrast enhanced CT and metabolic parameters may predict the presence of unilateral adrenal tumors to treat surgically through a practical two-parameter model. Most importantly, in a cost-effective scenario, we proposed a diagnostic flow-chart able to stratify patients into those to treat surgically versus those to follow-up only and addressing unilateral adrenal lesions with uncertain management to an [18F]FDG PET scan.

\section{Declarations}

\section{Ethics approval}

The study received approval from the Ethical Committee of the IRCCS Azienda Ospedaliero-Universitaria di Bologna (CE-AVEC), Italy.

\section{Funding}

The study was performed without any financial support and funding.

\section{Conflicts of interest}

The authors declare no conflicts of interest.

\section{Availability of data and material}

Data are available on request to the corresponding author.

\section{References}

1. Sherlock M, Scarsbrook A, Abbas A, Fraser S, Limumpornpetch P, Dineen R, et al. Adrenal Incidentaloma Endocrine Reviews. 2020;41:775-820.

2. Fassnacht M, Arlt W, Bancos I, Dralle H, Newell-Price J, Sahdev A, et al. Management of adrenal incidentalomas: European Society of Endocrinology Clinical Practice Guideline in collaboration with the European Network for the Study of Adrenal Tumors. Eur J Endocrinol. 2016;175:G1-34.

3. Dinnes J, Bancos I, Ferrante di Ruffano L, Chortis V, Davenport C, Bayliss S, et al. MANAGEMENT OF ENDOCRINE DISEASE: Imaging for the diagnosis of malignancy in incidentally discovered adrenal masses: a systematic review and meta-analysis. Eur J Endocrinol. 2016;175:R51-64.

4. Berland LL, Silverman SG, Gore RM, Mayo-Smith WW, Megibow AJ, Yee J, et al. Managing Incidental Findings on Abdominal CT: White Paper of the ACR Incidental Findings Committee. Journal of the American College of Radiology. 2010;7:754-73.

5. Woo S, Suh CH, Kim SY, Cho JY, Kim SH. Pheochromocytoma as a frequent false-positive in adrenal washout CT: A systematic review and meta-analysis. Eur Radiol. 2018;28:1027-36. 
6. Blake MA, Kalra MK, Maher MM, Sahani DV, Sweeney AT, Mueller PR, et al. Pheochromocytoma: an imaging chameleon. Radiographics. 2004;24(Suppl 1):87-99.

7. Sundin A. Imaging of Adrenal Masses with Emphasis on Adrenocortical Tumors. Theranostics. 2012;2:516-22.

8. Chen CC, Carrasquillo JA. Molecular imaging of adrenal neoplasms. J Surg Oncol. 2012;106:532-42.

9. Boland GWL, Blake MA, Hahn PF, Mayo-Smith WW. Incidental Adrenal Lesions: Principles, Techniques, and Algorithms for Imaging Characterization. Radiology. 2008;249:756-75.

10. Blake MA, Kalra MK, Sweeney AT, Lucey BC, Maher MM, Sahani DV, et al. Distinguishing benign from malignant adrenal masses: multi-detector row CT protocol with 10-minute delay. Radiology. 2006;238:578-85.

11. Kimura N, Takayanagi R, Takizawa N, Itagaki E, Katabami T, Kakoi N, et al. Pathological grading for predicting metastasis in phaeochromocytoma and paraganglioma. Endocr Relat Cancer. 2014;21:405-14.

12. Kim S-J, Lee S-W, Pak K, Kim I-J, Kim K. Diagnostic accuracy of 18F-FDG PET or PET/CT for the characterization of adrenal masses: a systematic review and meta-analysis. $\mathrm{Br} \mathrm{J}$ Radiol. 2018;91:20170520.

13. Tessonnier L, Sebag F, Palazzo FF, Colavolpe C, De Micco C, Mancini J, et al. Does 18F-FDG PET/CT add diagnostic accuracy in incidentally identified non-secreting adrenal tumours? Eur J Nucl Med Mol Imaging. 2008;35:2018-25.

14. Altinmakas E, Hobbs BP, Ye H, Grubbs EG, Perrier ND, Prieto VG, et al. Diagnostic performance of 18F-FDG-PET-CT in adrenal lesions using histopathology as reference standard. Abdom Radiol (NY). 2017;42:577-84.

15. Guerin C, Pattou F, Brunaud L, Lifante J-C, Mirallié E, Haissaguerre M, et al. Performance of 18F-FDG PET/CT in the Characterization of Adrenal Masses in Noncancer Patients: A Prospective Study. J Clin Endocrinol Metab. 2017;102:2465-72.

16. Vos EL, Grewal RK, Russo AE, Reidy-Lagunes D, Untch BR, Gavane SC, et al. Predicting malignancy in patients with adrenal tumors using 18 F-FDG-PET/CT SUVmax. J Surg Oncol. 2020;122:1821-6.

\section{Figures}


Patients with unilateral adrenal lesion who executed ceCT between 2007 and 2018

$$
(n=78)
$$

Patients who did not execute FDG-PET $(n=23)$

Excluded patients:

- executed FDG-PET earlier than CT $(n=2)$

- executed FDG-PET later than CT $(n=2)$ PET within 1 month from ceCT

$$
(n=51)
$$

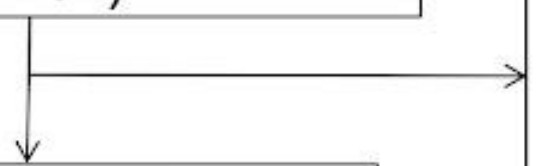

Patients with missing data on $\mathrm{ceCT}$ parameters $(n=3)$

Patients with complete data

$$
\text { ( } n=48)
$$

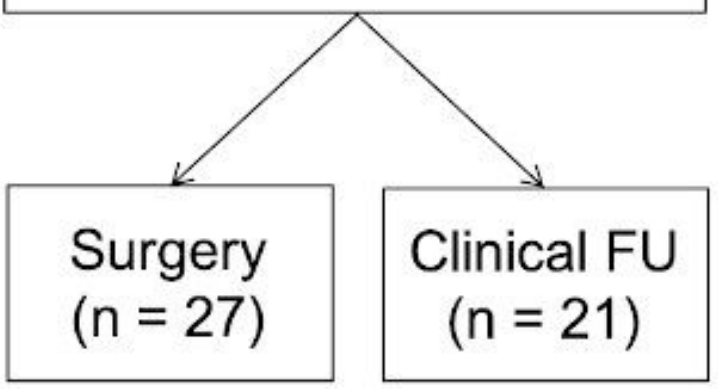

Figure 1

Consort diagram for patient selection. 


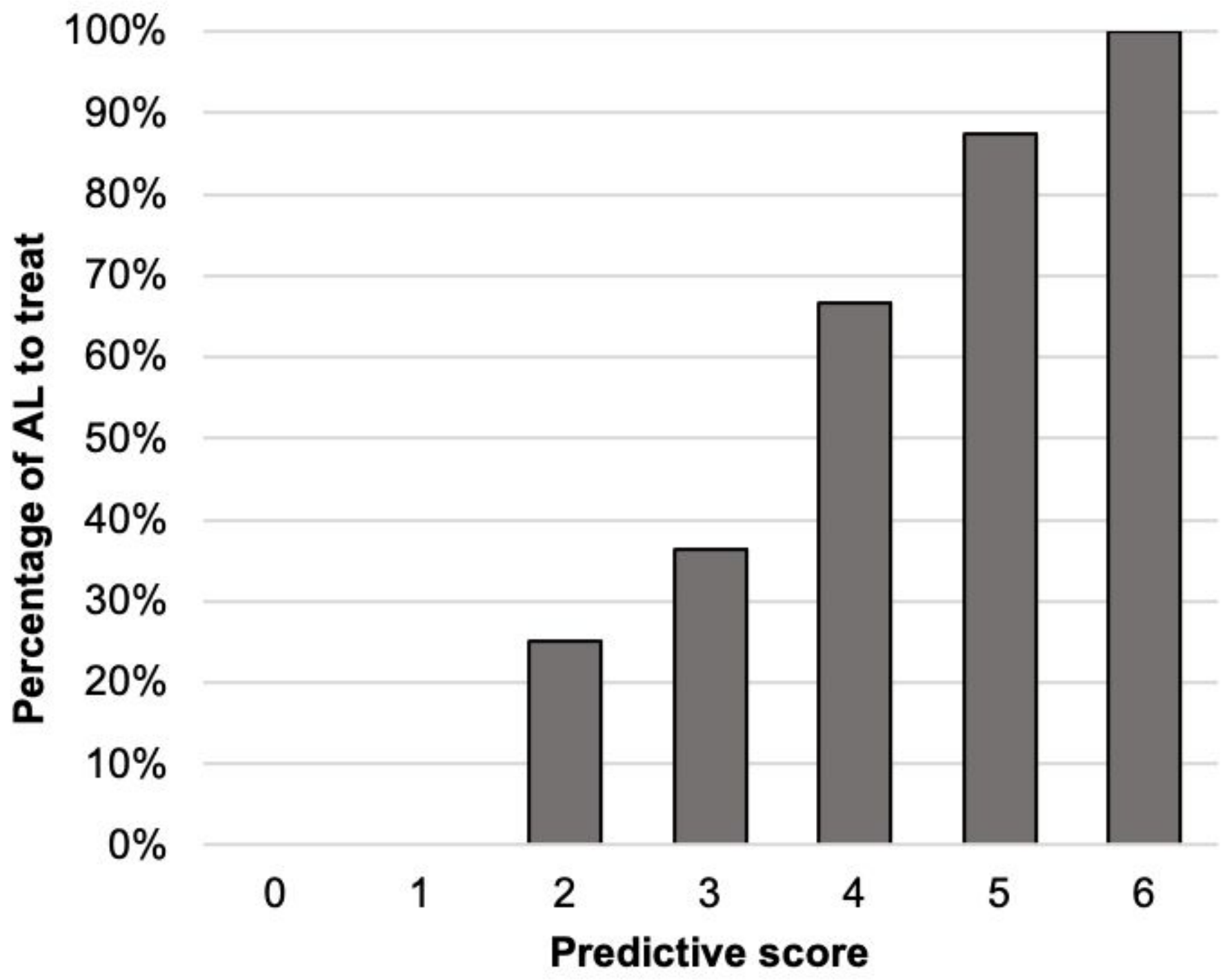

Figure 2

Barchart showing the percentage of AL-to-treat according to the imaging diagnostic score. 


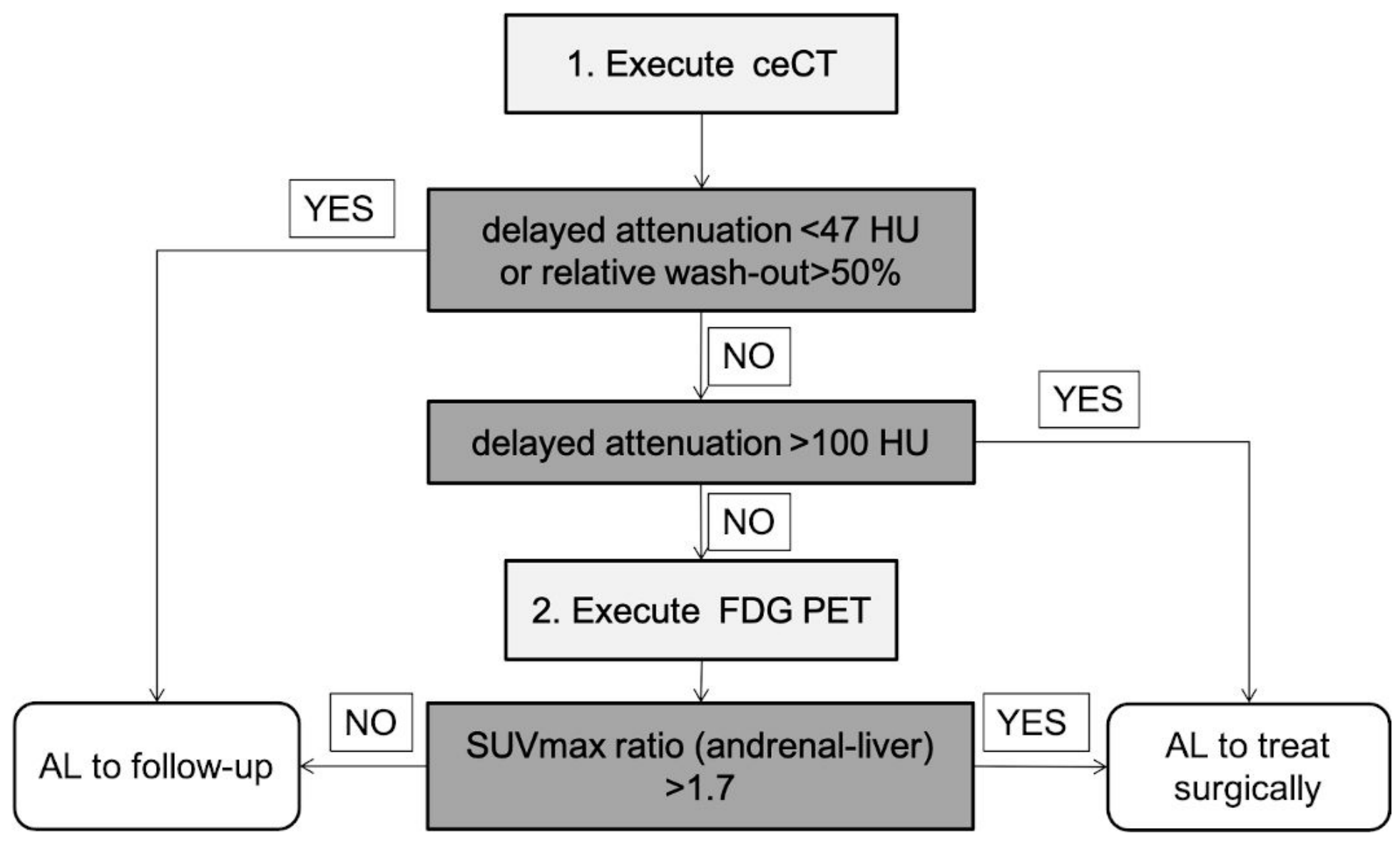

Figure 3

Diagnostic flow-chart for unilateral adrenal lesions.

\section{Supplementary Files}

This is a list of supplementary files associated with this preprint. Click to download.

- Supplementaryfile.docx 